\title{
Angiogenesis inhibitors revised and revived at AACR
}

The anticancer potential of angiogenesis inhibitors has been highly controversial. Clinical results of drugs such as endostatin and angiostatin have fallen short of expectations, and their value has been both hyped and decimated in news stories. Thus, a number of sessions at the 2002 American Association for Cancer Research (AACR) meeting in San Francisco last month were dedicated to establishing whether or not these drugs can really be used to treat cancer, and to the design of future trials. It seems that not enough was known about the molecular mechanisms of tumor angiogenesis when trials of antiangiogenic compounds began in the 1990s, and that the manner in which these drugs are administered must be changed to achieve maximum clinical efficacy.

There are currently 80 anti-angiogenic agents in clinical trials that have enrolled over 10,000 patients. The first cancer trials of anti-angiogenic agents were met with considerable anticipation, as these drugs had proven highly effective in treating cancer in mice. Dana-Farber and a group of affiliated hospitals began the first clinical study of endostatin in 1999. The first phase of the study was completed two years later, when doctors announced that patients receiving endostatin experienced few side effects and that tumor growth was stabilized.

But anti-angiogenic drugs had already been the topic of fierce public debate. In May 1998, their human potential was hyped in a New York Times article based on data from animal studies, which triggered some researchers to report their difficulties in reproducing experimental results in mice. Skepticism arose in scientific, clinical, pharmaceutical and financial circles, and cancer patients were confused by the conflicting information. Ultimately, the studies revealed that endostatin, as well as other inhibitors such as TNP-470, thalidomide, IM862, Neovastat, Angiozyme, and Combretastatin A4 Pro-drug, did cause tumor shrinkage in a few patients, although most patients experienced only tumor stabilization.

These results should have come as no surprise. Speaking at the AACR meeting, Michael O'Reilly, a researcher at the M.D. Anderson Cancer Center, reminded the audience that many years ago mouse model studies had shown that angiostatin had to be administered over the long term to in- duce tumor regression. "In humans, this translates into having to be treated for several months before you see an effect. But most patients don't have the luxury of waiting out this long period of tumor progression before regression occurs" said O'Reilly.

Other investigators revealed furthercaveats to using these drugs. According to Steve Libutti of the National Cancer Institute, the efficacy of anti-angiogenic agents "depends on the way in which you use them." For example, different tumors upregulate expression of different pro-angiogenic factors. Some express high levels of the pro-angiogenic molecule basic fibroblast growth factor (bFGF), so treating these cancers with drugs that target vascular endothelial growth factor (VEGF) is not

\section{American Association for Cancer Research}

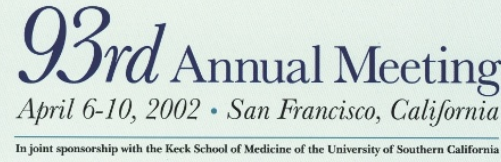

likely to be effective. In fact, Doug Hanahan of the University of California San Francisco pointed out that some tumors, such as pancreatic tumors, express up to eight different pro-angiogenic factors. Furthermore, tumors are also highly heterogeneous and comprise different cell types that express different combinations of factors.

Hanahan showed data to suggest that the timing with which an anti-angiogenic agent is delivered is crucial for efficacy. He compared the ability of different anti-angiogenic drugs to act at different stages of tumor development, such as preventing initial tumor growth, intervening in expansion or inducing regression. Anti-angiogenic drugs such as endostatin, angiostatin and SU5416 are most effective in preventing initial tumor development, whereas SU6668 is more effective at later stages of tumor development and induces regression.

Many of these drugs were also moved into large clinical trials too quickly, according to Roy Herbst of the M.D. Anderson Cancer Center. After the drugs were proven to be safe in Phase I trials, they went directly into larger trials before the biologically effective dose, pharmacokinetics or long-term effects of drug treatment had been well established. "We were essentially going to the clinic and doing the preclinical trials at the same time," said Herbst.

It has been difficult to determine the clinical efficacy of the compounds due to the lack of good endpoints that show whether or not an agent is working. "Just because the tumor doesn't decrease in size doesn't mean that the drug isn't workingtumors are highly heterogeneous," Libutti reminded the audience. Thus, a recent focus of angiogenesis research has been the development of clinical endpoints, such as imaging studies that measure reductions in tumor vasculature, or serum-based markers of vascular growth.

New findings about the source of cells that make up the tumor vasculature are also likely to affect therapy. Shahin Rafii's group at the Memorial Sloan-Kettering Cancer Center, New York, has shown that bone marrow-derived endothelial precursors are required for tumor angiogenesis (Nature Med. 7, 1194; 2001), a finding that changed many ideas about how tumors induce blood-vessel formation. "Researchers had thought for the past 30 years that tumors recruit all their vascular endothelial cells locally," said Judah Folkman, of Harvard University. Because Rafii showed that some tumors recruit local endothelial cells, whereas others recruit bone-marrow endothelial precursors, "Chemotherapy, which destroys the bone marrow, might therefore partially slow tumor development by inhibiting angiogenesis. So it will be important to find out if when you replace the bone marrow in cancer patients, you are actually redelivering cells that can support the tumor vasculature," said Folkman.

Several published studies have shown that anti-angiogenic agents act synergistically with chemotherapy or radiation therapy. For example, radiotherapy can be used to destroy a primary tumor, but combined treatment with angiostatin has been shown to suppress metastases. As chemotherapy has also been shown to upregulate tumor expression of VEGF, the combination of chemotherapy and antiVEGF drugs is likely to be effective. So far, 12 drugs have been developed to specifically target VEGF-receptor signaling. "Combination therapy will overcome many of the limitations of individual anticancer agents," said O'Reilly.

Kristine Novak, San Francisco 